\title{
MINIMAL RESOLUTIONS AND OTHER MINIMAL MODELS
}

\author{
Agustí RoIg
}

\begin{abstract}
In many situations, minimal models are used as representatives of homotopy types. In this paper we state this fact as an equiv alence of categories. This equivalence follows from an axiomatic definition of minimal objects. We see that this definition includes examples such as minimal resolutions of Eilenberg-NakayamaTate, minimal fiber spaces of Kan and $\Lambda$-minimal $\Lambda$-extensions of Halperin. For the first one, this is done by generalizing the construction of minimal resolutions of modules to complexes. The others follow by a caracterization of minimal objects in bifibred categories.
\end{abstract}

\section{Introduction}

Minimal models appear in different situations as representative of homotopy types. We claim that this is an intrinsic property of those objects called "minimal", and does not depend on the particular construction employed in cach category. To this end, wc propose an axiomatic definition of minimality, inspired by the definition of minimal $(R, r)$-algebras of $[\mathrm{H}-\mathrm{T}]$. Our result is that the homotopy category of a model category and the category of its minimal objects and homotopy classes of maps are equivalent (Theorem 1.17).

We also prove that our axiomatic definition produces the objects that are commonly known as "minimals". In particular, we see that the minimal resolutions of Eilenberg-Nakayama-Tate are minimal objects of a category of cochain complexes. This is done by generalizing the construction of these minimal resolutions of modules to complexes; i.e., graded modules with differential (Theorems 2.4 and 2.5). We also verify that minimal fibre spaces of [Kan] and $\Lambda$-minimal $\Lambda$-extensions of [Hal] are minimal objects of bifibred categories (see [Roig1]) in terms of our definition (Theorem 3.2). Other examples may be found in [Roig2] and [Roig3]. 
I am grateful to Francisco Guillén, Vicente Navarro Aznar, Pere Pascual-Gainza and Daniel Tanré for helpful discussions and suggestions. I am also grateful to the referee for his remarks and criticisms.

\section{Minimal objects and the homotopy category}

Let $\mathcal{C}$ be a category and $S \subset \operatorname{mor} \mathcal{C}$ a class of morphisms of $\mathcal{C}$. We will often denote the fact that a morphism $s: a \rightarrow b$ of $\mathcal{C}$ is in $S$ by $s: a \stackrel{\sim}{\rightarrow} b$ and we will loosely say that $s$ is a $S$-quasi-isomorphism (quism) and $a$ is $S$-quasi-isomorphic to $b$. We will also say that $a$ is a $S$-left model for $b$ or that $b$ is a $S$-right model for $a$.

Definition 1.1. An object $m$ of $\mathcal{C}$ is $S$-left minimal if, for all $s$ : $x \rightarrow m \in S$, there exists a section $s^{\prime}: m \rightarrow x$; i.e., $s s^{\prime}=1_{m}$. A $S$-left minimal model of an object $a \in \operatorname{obj} \mathcal{C}$ is a $S$-left model $m \rightarrow a$ with $m$ a left minimal object.

By inverting arrows in the previous definition we have the notions of $S$-right minimal object and $S$-right minimal model (see [Roig3]). From now on we suppose that the class $S$ is fixed for every category and consider only the "left part" of the theory, unless otherwise stated. So we will simply say minimal object, model and minimal model. The relative version of definition 1.1 is the following (cf. [B-G], [Hal] and $[\mathbf{H}-\mathbf{T}]$ ): let $\mathcal{C}$ be a category; $a$ an object of $\mathcal{C}$ and $S \subset$ mor $\mathcal{C}$. Let $a \backslash S$ be the class of morphisms of the category of objects under $a, a \backslash \mathcal{C}$, made up by the commutative triangles of $\mathcal{C}$

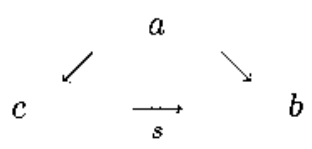

in which $s \in S$.

Definition 1.2. A morphism $a \rightarrow b$ of $\mathcal{C}$ is a minimal morphism if it is a $a \backslash S$-minimal object of $a \backslash \mathcal{C}$.

For the first results, it is necessary that the class $S$ verifies the

\section{Hypothesis 1.3.}

(i) isomorphisms of $\mathcal{C}$ are in $S$, and

(ii) if in the diagram of $\mathcal{C}, x \stackrel{f}{\rightarrow} y \stackrel{g}{\rightarrow} z$, two of the morphisms $\{f, g, g f\}$ are in $S$, then so is the third. 
These hypothesis are fulfilled if, for instance, $\mathcal{C}$ is a model category and $S=$ we is the class of its weak equivalences or if $S$ is the class of morphisms made invertible by some functor $H: \mathcal{C} \rightarrow \mathcal{D}$. Then we can easily prove (see [Roig3]).

Proposition 1.4. If $m$ and $n$ are minimal objects and $s: m \rightarrow n$ is a quism, then $s$ is an isomorphism.

Definition 1.5. Let $\mathcal{C}$ be a category with an initial object $e$. An object $x$ of $\mathcal{C}$ is acyclic if it is quasi-isomorphic to $e$.

Corollary. An object $x$ of $\mathcal{C}$ is minimal and acyclic if and only if it is initial.

Proof: An initial object $e$ is always minimal and, since $1_{e}$ is in $S$ by $1.3(\mathrm{i})$, it is also acyclic. On the other hand if $x$ is minimal and acyclic there is a morphism in $S x \rightarrow e$ which is, by 1.4, an isomorphism.

The following is a useful criterium for minimality.

Proposition 1.6. If $\mathcal{C}$ admits a class of objects $\mathcal{M}$ such that:

(a) every object has a model in $\mathcal{M}$, and

(b) every morphism of $S$ between objects of $\mathcal{M}$ is an isomorphism, then

(1) the objects of $\mathcal{M}$ are minimal, and

(2) every minimal object of $\mathcal{C}$ is isomorphic to an object of $\mathcal{M}$.

Proof: Sce [Roig3].

Definition 1.7. If $\mathcal{C}$ has a class of objects $\mathcal{M}$ that fulfiles conditions (a) and (b) of Proposition 1.6, we will say that $\mathcal{C}$ has enough minimals.

We will denote by $\mathrm{HoC}$ the homotopy category; i.e, the category obtained from $\mathcal{C}$ by adjoining the inverses of the morphisms in $S$ (sce [Qui]). It is also called the localized category, $\mathcal{C}_{S}([\mathrm{G}-\mathrm{Z}])$, or the derived category, in the case where $\mathcal{C}$ is the category of complexes of an abelian category ([Hart]). If two objects of $\mathcal{C}$ are isomorphic in HoC they are said "to have the same homotopy type". For the moment we will assume also

Hypothesis 1.8. $S$ admits a calculus of right fractions.

This is the case when $\mathcal{D}$ is a model category with only fibrant objects, $\mathcal{C}=\pi \mathcal{D}$ being the category obtained identifying homotopic maps of 
$\mathcal{D}$ and $S \subset \operatorname{mor} \mathcal{C}$ being the image of the weak equivalences of $\mathcal{D}$ by the projection functor $\mathcal{D} \rightarrow \pi \mathcal{D}$. We will develop our results under hypothesis 1.8 and then restate them "up to homotopy" for a model category. Finally, in our particular examples, all objects will be fibrant.

When $S$ admits a calculus of right fractions, the morphisms of HoC from $a$ to $b$ can be represented by sequences or morphisms of $\mathcal{C}$ as

$$
a \stackrel{\sim}{\sim} \longrightarrow b
$$

and the isomorphisms are exactly those sequences where both morphisms of (1) are in $S$. Then, with this hypothesis, two minimal objects of $\mathcal{C}$ which are isomorphic in $\mathrm{HoC}$ are necessarity isomorphic in $\mathcal{C}$ : If in (1) $a$ and $b$ are minimal, we have a section of $a \leftarrow$. which is in $S$ by (i) and (ii) of 1.3. Hence, its composition with $\rightarrow b$ is also in $S$. Thus we have a quism between minimal objects which, by 1.4 , is an isomorphism. Therefore, homotopy types become isomorphism classes when we use minimal objects. We are going to state this fact as an equivalence of categories. To begin with, we have a lifting property.

Proposition 1.9. Let $\rho: a \stackrel{\sim}{\rightarrow} b \in S$ and $f: m \rightarrow b$ a morphism with $m$ minimal. Then there exists a unique $\bar{f}: m \rightarrow$ a such that $\rho \bar{f}=f$.

Proof: Because of the propcrties of the calculus of fractions, for every diagram in $\mathcal{C}$,

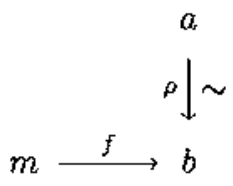

there exists morphisms $\rho^{\prime}: x \stackrel{\sim}{\rightarrow}$ and $f^{\prime}: x \rightarrow a$ such that $\rho f^{\prime}=f \rho^{\prime}$. Then, as $m$ is minimal, $\rho^{\prime}$ has a section $s: m \rightarrow x$. Take $\bar{f}=f^{\prime} s$ and so $\rho \bar{f}=\rho f^{\prime} s=f \rho^{\prime} s=f$.

Secondly, let $g: m \rightarrow a$ be another morphism such that $\rho g=f$. Then $\rho \bar{f}=\rho g$. But $\rho \in S$, and so, by the calculus of fractions, there exists $r: z \rightarrow m \in S$ such that $\bar{f} r=g r$ and because $m$ is minimal, this morphism has a section $s: m \rightarrow z, r s=1_{m n}$. So $g=g r s=\bar{f} r s=\bar{f}$.

Corollary. Two minimal models of an object a are isomorphic by a unique isomorphism of $\mathrm{C} / \mathrm{a}$.

The next proposition will allow us to define the "minimal model functor", when we have enough minimals. 
Proposition 1.10. Let $f: a \rightarrow b$ be a morphism of $\mathcal{C}$ and $\rho_{a}: m_{\mathfrak{a}} \rightarrow$ $a$ and $\rho_{b}: m_{b} \rightarrow b$ two minimal models. Then there exists a unique morphism $m_{f}: m_{a} \rightarrow m_{b}$, that renders commutative the diagram

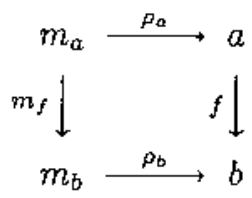

Proof: Applying the calculus of fractions two times, we have a commutative diagram

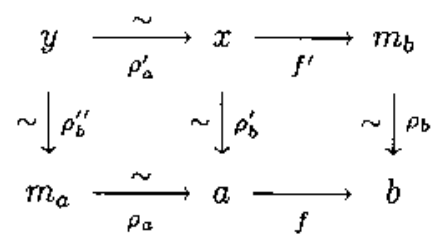

So, by the minimality of $m_{a}$, we have $s: m_{a} \rightarrow y$ such that $\rho_{b}^{\prime \prime} s=1_{m_{a}}$. We let $m_{f}=f^{\prime} \rho_{a}^{\prime} s$ and have $\rho_{b} m_{f}=\rho_{b} f^{\prime} \rho_{a}^{\prime} s=f \rho_{b}^{\prime} \rho_{a}^{\prime} s=f \rho_{a} \rho_{b}^{\prime \prime} s=f \rho_{a}$.

Let $\varphi: m_{a} \rightarrow m_{b}$ be another morphism that makes commutative diagram (2): $\rho_{b} \varphi=f \rho_{a}=\rho_{b} m_{f}$. Then, because $\rho_{b} \in S$, by the calculus of fractions, there exists $r: z \rightarrow m_{a} \in S$ such that $\varphi r=m_{f} r$ and, because $m_{a}$ is minimal, we have a section $s: m_{a} \rightarrow z$ of $r$. So, $\varphi=$ $\varphi r s=m_{f} r s=m_{f}$.

Remark 1.11. $m_{f}$ is not necessarily the minimal model of $f$ in the sense of definition 1.2. For instance, unless $a$ is a minimal object, $m_{f}$ needs not to be an object of $a \backslash \mathcal{C}$.

We have not assumed yet the existence of a minimal model for every object. Let us suppose now that $\mathcal{C}$ has enough minimals: for every $a \in \operatorname{obj} \mathcal{C}$, we choose a minimal model which will be denoted by $\rho_{a}$ : $M(a) \rightarrow a$. Let us also note by $\mathcal{C}_{m}$ (or $\mathcal{C}_{\min }$ when we will talk about bifibred categories) the full subcategory of minimal objects. Then we have

Corollary. The choice of a minimal model defines a functor

$$
M: \mathcal{C} \longrightarrow \mathcal{C}_{m},
$$

by $M(a)=m_{a}$ and $M(f)=m_{f} ;$ and a morphism of functors

$$
\rho: M \longrightarrow 1_{\mathcal{C}}
$$


by $\rho_{a}: m_{a} \rightarrow a$.

Proof: Although there are different choices for $M(a)$ and $M(f)$, the unicity of Propositions 1.9 and 1.10 makes the correspondence functorial.

From what we have seen, it is clear that the functor $M$ carries morphisms of $S$ into isomorphisms of $\mathcal{C}_{m}$. In fact, if in (2) $f \in S$, then $f \rho_{a}: m_{a} \rightarrow b$ is a minimal model of $b$. Hence, there exists an isomorphism of $C / b, \varphi: m_{u} \rightarrow m_{b}$, which, because of the uniqueness is equal to $m_{f}$. Therefore, $M$ factorizes in a unique fashion through the localization functor $\gamma: \mathcal{C} \rightarrow \mathcal{C}_{S}$. That is to say, we have the commutative diagram of functors

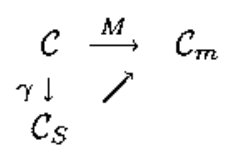

We will note also by $M$ this factorization and call it the minimal model functor.

Theorem 1.12. The functor $M: \mathcal{C}_{S} \rightarrow \mathcal{C}_{m}$ is an equivalence of categories.

Proof: Let $i: \mathcal{C}_{m} \rightarrow \mathcal{C}_{S}$ be the composition of the inclusion $\mathcal{C}_{m} \rightarrow \mathcal{C}$ and the localization $\mathcal{C} \rightarrow \mathcal{C}_{S}$. We will show that $\ell$ is an cquivalence, quasi-inverse of $M$, by defining isomorphisms $\eta: 1_{\mathcal{C}_{m}} \rightarrow M_{l}$ and $\varepsilon$ : $\iota M \rightarrow 1_{C_{S}}$. Let $m$ be minimal. Then, by corollary of Proposition 1.9 , there exists an isomorphism $\eta_{m}: m \rightarrow M \iota(m)$. On the other hand, define $\varepsilon_{a}: \iota M(a) \rightarrow a$ as the isomorphism in $\mathcal{C}_{S}$ induced by the choice in $\mathcal{C}$ of the minimal model of $a, M(a) \rightarrow a$. It is easy to check that these definitions are natural in $m$ and $a$, respectively.

Remark 1.13. We can easily dualize the previous results taking into account that Hypothesis 1.3 are self-duals and rcplacing the existence of calculus of right fractions by that of left fractions.

As we have said, the existence of a calculus of right fractions is fullfiled if we are working in a model category. So, from now on, we will assume, instead of Hypothesis 1.3 and 1.8,

Hypothesis 1.14. $\mathcal{C}$ is a model category, $S=$ we is the class of its weak equivalences and all the objects of $\mathcal{C}$ are fibrant.

Let us recall that, as a consequence, we have properties (i) and (ii) of Hypothesis 1.3 and a calculus of fractions in $\pi C$. To begin with the 
translation of previous results in this setting, we have a relative version of 1.9 .

Proposition 1.15. Let

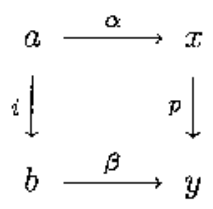

be a commutative diagram of $\mathcal{C}$ in which $a$ is a minimal object and $i$ is a minimal morphism. Then, if $p$ is a weak equivalence, there exists $\bar{\beta}: b \rightarrow x$, unique up to homotopies, such that $p \bar{\beta} \sim \beta$ and $\tilde{\beta} i \sim \alpha$.

Proof: Let us factorize $p$ in a cofibration $j: x \rightarrow z$ and a fibration $q: z \rightarrow y$, both trivial ones. Take the pull-back of $\beta$ and $q$ :

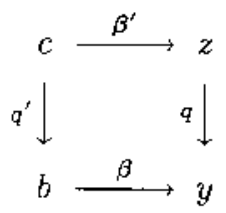

Trivial fibrations are stable under pull-backs, so $q^{\prime}$ is a trivial fibration, which we can consider a trivial fibration of $a \backslash \mathcal{C}$ from the morphism induced by $j \alpha$ and $i, \gamma: a \rightarrow c$, to $i$. But $i$ is a minimal morphism, so there exists $s: b \rightarrow c$ such that $q^{\prime} s=1_{b}$ and $s i=\gamma$. On the other hand, $j$ is a trivial cofibratrion: so it has a homotopic inverse $r: z \rightarrow x$. Then, $\tilde{\beta}=r \beta^{\prime} s$ is the lifting we are looking for: $\tilde{\beta} i=r \beta^{\prime} s i=r \beta^{\prime} \gamma=r j \alpha \sim \alpha$ and $p \vec{\beta}=q j r \beta^{\prime} s \sim q \beta^{\prime} s=\beta q^{\prime} s=\beta$.

The uniqueness up to homotopy of $\tilde{\beta}$ is also an easy verification.

Corollary 1. Let $\rho: a \stackrel{\sim}{\rightarrow} b \in$ we and $f: m \rightarrow b$ be a morphism with $m$ a minimal object. Then there exists $\bar{f}: m \rightarrow a$, unique up to homotopies, such that $\rho \bar{f} \sim f$.

Proof: Take $a=e$ in the previous proposition.

Corollary 2. Two minimal models of $a \in \mathcal{C}$ are isomorphic. The isomorphism is unique up to homotopies of $\mathcal{C} / a$.

The rest of the results admit analogous modifications. 
Proposition 1.16. Let $f: a \rightarrow b$ be a morphism of $\mathcal{C}$ and $\rho_{a}: m_{a} \rightarrow a$ and $\rho_{b}: m_{b} \rightarrow b$ two minimal models. Then there exists a morphism $m_{f}: m_{a} \rightarrow m_{b}$, unique up to homotopies, that renders commutative up to homotopy the diagram

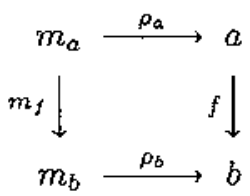

Let us assume that $\mathcal{C}$ has enough minimals. Given $a \in$ obj $\mathcal{C}$, let us choose a minimal model: $\rho_{a}: M(a) \rightarrow a$. Since previous results are stated "up to homotopy", the correspondence $a \mapsto M(a)$ is not necessarily functorial and the weak equivalences $p_{a}$ do not necessarely define a morphism of functors $M \rightarrow I_{\mathcal{C}}$. Nevertheless, we have a well-defined functor and a morphism of functors if we take as target the category $\pi \mathcal{C}_{m}$, which has as objects the minimal ones of $\mathcal{C}$ and as morphisms the homotopy classes of morphisms of $\mathcal{C}$.

Corollary. The choice of a minimal model defines a functor

$$
M: \mathcal{C} \longrightarrow \pi \mathcal{C}_{m}
$$

by $M(a)=m_{a}$ and $M(f)=m_{f}$, and a morphism of functors

$$
\rho: M \longrightarrow 1_{\pi C}
$$

by $\rho_{a}: m_{a} \rightarrow a$.

We also have a unique factorization of $M$ through the localization functor and Theorem 1.12 now reads:

Theorem 1.17. The functor $M: \mathrm{HoC} \rightarrow \pi \mathcal{C}_{m}$ is an equivalence of categories.

Remarks 1.18. (1) All the above facts are independent of the classes of fibrations and cofibrations we choose. So, for a given class of weak equivalences, all the possible model structures share one class of cofibrant objects in common, if they exist: the minimal ones.

(2) The last theorem could also be deduced from the fact that minimal morphisms are necessarily cofibrations (in a closed model category, at least) and then applying [Qui, Theorem 1', Section 1, chapter I], taking into account that we do not need oll the cofibrant objects, but only one representative for each homotopy type (for instance, a minimal one).

(3) In order to dualize previous results, we need only to substitute cofibration for fibration and vice-versa. 


\section{Minimal resolutions of complexes}

Let $R$ be a unitary commutative ring. A $R$ - $d g$ module is a graded module $M$ with a differential of degree +1 ; i.e., a cochain complex. Let us take as $S$ the class of quasi-isomorphisms; that is to say, the morphisms which induce an isomorphism in cohomology. Before we restrict ourselves to local rings, let us examine some examples produced by our definition.

Proposition 2.1. Let $M$ be a $R$-dg module with zero differential. Then $M$ is minimal if and only if $M^{i}$ is a projective module for each $i$.

Proof: Let $M$ be a cochain complex, with a projective module in each degree, zero differential and $X \stackrel{\sim}{\rightarrow} M$ a quism. Then, for every $i$, we can choose a section of $Z^{i} X \rightarrow H^{i} X \cong M^{i}$. These give us a morphism of $R$-dg modules $M \rightarrow X$ which is a section of the quism above.

Reciprocaly, assume that $M$ is minimal and let $f: X \rightarrow M^{i}$ be an epimorphism of $R$-modules. Consider the morphism of $R$-dg modules

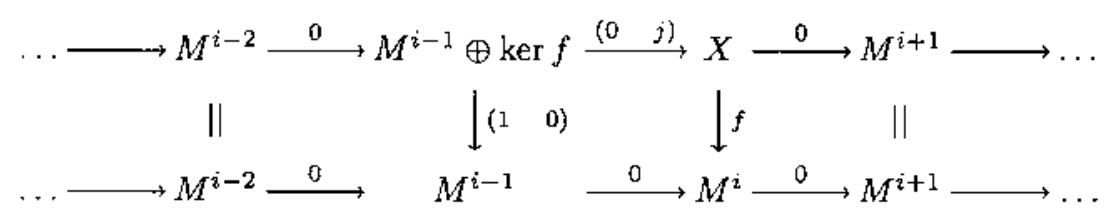

(where the horizontal arrows are the differentials and $j: \operatorname{ker} f \hookrightarrow X$ is the inclusion). Obviously, it is a quism and, since $M$ is minimal, it has a section. In particular, $f$ has a section and so $M^{i}$ is a projective $R$-module.

Corollary. Let $M$ be a $R$-module, considered as a homogeneous $d g$ module with zero differential. Then $M$ is minimal if and only if it is projective.

Proposition 2.2. Let $M$ be a $R$-dg module such that $H^{i} M$ is a projective $R$-module for each $i$. Then $H M$ is a minimal model of $M$.

Proof: Let $Z^{i} M \rightarrow H^{i} M$ be the natural projection and $s^{i}: H^{i} M \rightarrow$ $Z^{2} M$ a section. Then $s=\left(s^{i}\right): H M \rightarrow M$ is a quism of $R$-dg modules and so $H M$ is a model for $M$. Becausc of Proposition 2.l it is a minimal model.

Corollary 1. If $R$ has zero global dimension, then:

(1) Every $R$-dg module has a minimal model.

(2) $M$ is minimal if and only if $M$ has zero differential. 
Corollary 2. If $H^{i} M$ is projective for each $i$, then

$$
\operatorname{Tor}_{R}(M, N)=H M \otimes_{R} H N
$$

This is the case, for instance, if $R$ is a semi-simple ring (e.g., a field or a group algebra $\mathbf{k}[G]$, where $G$ is a finite abelian group and the characteristic of $\mathrm{k}$ does not divide the order of $G$ ). Apart from this, minimal projective resolutions of modulcs arc known to exist when $R$ is a noetherian local ring ([Eil], [Tate]). We are going to show that these rcsolutions are minimal objects of an appropiate category of dg modules. To do this we will generalize the construction of minimal resolutions in order to obtain models of graded modules with differential. So, let $R$ be a. noetherian local ring, $m$ its maximal ideal and $\mathrm{k}=R / m$ its residue field.

Definition 2.3. We will say that $M$ is a ENT-minimal complex if

(1) $M^{i}$ is a free $R$-module for every $i$, and

(2) $d M \subseteq m M$.

For instance, if we have

$$
\cdots \longrightarrow M^{-2} \longrightarrow M^{-1} \longrightarrow M^{0} \longrightarrow X \longrightarrow 0
$$

with $X$ a $R$-module and $M$ a ENT-minimal complex acyclic except in dimension zero which is $H^{0} M \cong X$, then $M$ is a minimal resolution of the $R$-module $X$ in the sense of [Eil] and [Tate]. This kind of projective resolutions have the property that $M \otimes_{R} \mathrm{k}$ has zero differential. Hence $\operatorname{Tor}_{R}^{i}(\mathrm{k}, \mathrm{k})=M^{i} / m M^{i}$.

The following theorems show, in particular, that in the category $\operatorname{Mdg}_{f g}^{-}(R)$, of $R$-dg modulcs bounded above and finitely generated in each degree, the ENT-minimal complexes are the minimal objects in the sense of our Definition 1.1.

Theorem 2.4. Let $f: L \rightarrow M$ be a quism between ENT-minimal complexes bounded above and finitely generated in each degree. Then $f$ is an isomorphism.

Theorem 2.5. Each $R$-dg module with bounded above and finite type cohomology has a ENT-minimal model bounded above and finitely generated in each degree.

Corollary 1. ENT-minimal complexes are the minimal objects of $\operatorname{Mdg}_{f g}^{-}(R)$.

Proof: This follows from the previous thcorems and Proposition 1.6. 
Corollary 2. The inclusion $\operatorname{Mdg}_{f g}^{-}(R)_{m} \hookrightarrow \operatorname{Mdg}_{f g}^{-}(R)$ induces an equivalence of categories

$$
\pi \mathrm{Mdg}_{f_{g}}^{-}(R)_{m} \cong \mathrm{HoMdg}_{f_{g}}^{-}(R)
$$

Proof: This follows from the previous corollary and Theorem 1.17.

Remark 2.6. The hypothesis of bounded cohomology is not enough for Theorem 2.4, as may be seen in the next example.

Example 2.7. Consider the unbounded complex of $Z /(4)$-modules

$$
\cdots \longrightarrow \mathrm{Z} /(4) \stackrel{2}{\longrightarrow} \mathrm{Z} /(4) \stackrel{2}{\longrightarrow} \mathrm{Z} /(4) \longrightarrow \ldots
$$

It is ENT-minimal and acyclic (so it has bounded cohomology), but it is not isomorphic to the null complex (cf. Corollary to 1.4).

Proof of Theorem 2.4: The proof is not the same as that of the comparison theorem for minimal resolutions because our complexes are not acyclic (sce [Mat]).

We may assume that $L^{i}=M^{i}=0$ for every $i>0$. By hypothesis, in the diagram

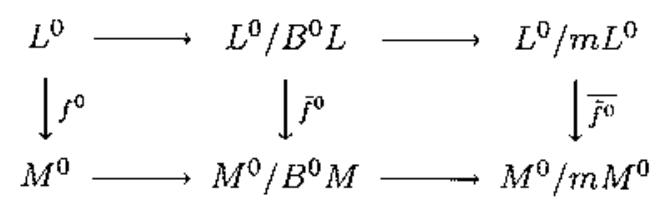

the middle column is an isomorphism of $R$-modules and, as a consequence, the right one $\overline{\hat{f}^{0}}=f^{0} \otimes_{R} \mathrm{k}=\tilde{f}^{0} \otimes_{R} \mathrm{k}$, is an isomorphism of finite dimensional k-vectorial spaces. $L^{0}$ and $M^{0}$ are free and finitely generated, so $f^{0}$ is defined by a square matrix $T$ with $\operatorname{det} T \notin m$. Hence $f^{0}$ is an isomorphism.

For $f^{i}$ with $i<0$, showing that

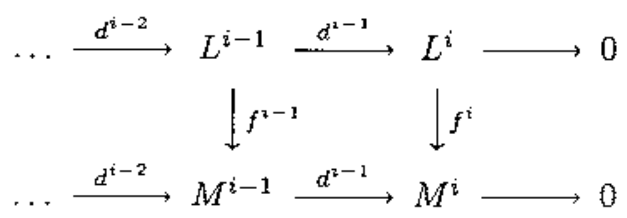

is a quism and applying the argument employed for $i=0$, enables us to conclude that $f^{i}$ is an isomorphism of $R$-modules. Let us assume that:

$(\mathrm{a})_{i+1} f^{i+1}: L^{i+1} \rightarrow M^{i+1}$, and

(b) $i+1 \quad \tilde{f}^{i+1}: L^{i+1} / B^{i+1} L \rightarrow M^{i+1} / B^{i+1} M$ 
are isomorphisms of $R$-modules. In degrees $j<i, H^{j} f$ is an isomorphism by hypothesis. In degree $i$, we have the exact scquences

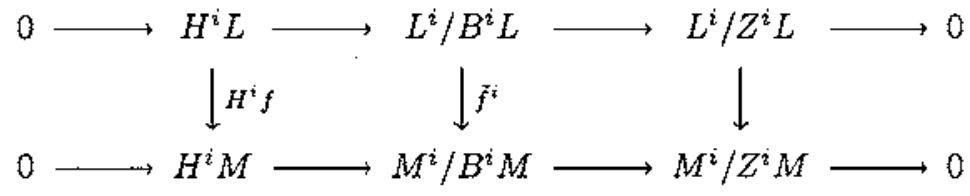

The first column is an isomorphism of $R$-modules by hypothesis. The third one is nothing but $f^{i+1}: B^{i+1} L \rightarrow B^{i+1} M$. Hence, we only need to check that $f_{\mid B^{2+1} L}^{i+1}$ is and isomorphism. And this follows from the diagram

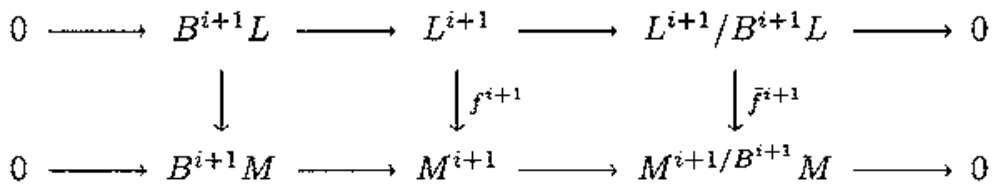

in which rows are exact and the second and the third columns are isomorphisms by hypothesis.

Proof of the Theorem 2.5: Let $X$ be a $R$-dg module with bounded above and finite type cohomology. We may assume, for instance, $H^{i} X=$ 0 for all $i>0$. Then the subcomplex $\tau_{\leq-1} X \hookrightarrow X$

$$
\left(\tau_{\leq-1} X\right)^{i}= \begin{cases}X^{i} & \text { if } i<0 \\ Z^{0} X & \text { if } i=0 \\ 0 & \text { if } i>0\end{cases}
$$

is quasi-isomorphic to $X$ and bounded above itself. Hence, we may assume that $X^{i}=0$ for all $i>0$. We will make use of the following lemma in our construction of the ENT-minimal model of $X$ (see [Mat]):

Lemma. Let $X$ be a finitely generated $R$-module, $L$ a free $R$-module over a minimal base of $X$ and $\varepsilon: L \rightarrow X$ the canonical morphism. Then $\operatorname{ker} \varepsilon \subseteq m L$.

Let $M^{0}$ be a free $R$-module over a minimal base of $H^{0} X$ and $\varepsilon: M^{0} \rightarrow$ $H^{0} X$ the canonical morphism. We define $M(0)$ to be the $R$-dg module

$$
M(0)^{i}= \begin{cases}M^{0} & \text { if } i=0 \\ 0 & \text { otherwise }\end{cases}
$$


Then, the morphism of complexes $s(0): M(0) \rightarrow X$,

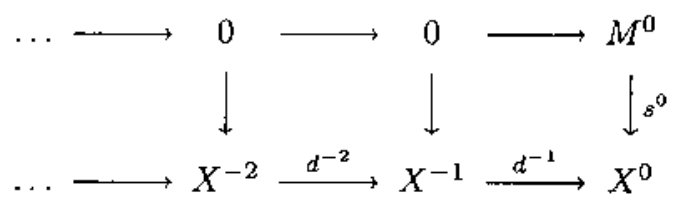

where $s^{0}: M^{0} \rightarrow Z^{0} X$ is a lifting of $\varepsilon$, verifies:

(a) $M(0)$ is ENT-minimal and finitcly generated in each degree,

(b) $s_{*}^{i}$ is an isomorphism for every $i>0$ and an epimorphism for $i=0$, and

(c) $)_{0}$ ker $s_{*}^{0} \subseteq m M^{0}$, because of the lemma.

Assume we have constructed a $R$-dg module $M(p+1)=\left\{M^{i}\right\}, M^{i}=0$ for $i<p+1$ and a morphism of $R$-dg modules $s(p+1): M(p+1) \rightarrow$ $X, s(p+1)=\left\{s^{i}\right\}, s^{i}=0$ for $i<p+1$,

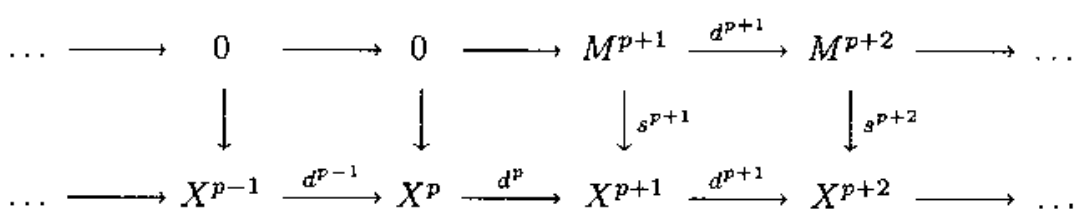

in such a way that

$(a)_{p+1} M(p+1)$ is ENT-minimal and finitely generated in each degree,

(b) $p+1 s_{*}^{i}$ is an isomorphism for every $i>p+1$ and an epimorphism for $i=p+1$, and

(c) $p+1$ ker $s_{*}^{p+1} \subseteq m M^{p+1}$.

Then, take

(1) $L_{1}^{p}$ a free $R$-module over a minimal base of ker $s_{*}^{p+1}$ and $\varepsilon_{1}$ : $L_{1}^{p} \rightarrow \operatorname{ker} s_{*}^{p+1}$ the canonical morphism. Since $M^{p+1}$ and $H^{p+1} X$ are finitely generated, and $R$ is noetherian, $L_{1}^{p}$ is also finitely generated,

(2) $d_{1}^{p}$ the composition $L_{1}^{p} \stackrel{\varepsilon_{1}}{\rightarrow} \operatorname{ker} s_{*}^{p+1} \hookrightarrow H^{p+1} M \hookrightarrow M^{p+1}$,

(3) $s_{1}^{p}: L_{1}^{p} \rightarrow X^{p}$ a lifting of $s^{p+1} d_{1}^{p}: L_{1}^{p} \rightarrow \operatorname{im} d^{p}$,

(4) $L_{2}^{p}$ a free $R$-module over a minimal base of coker $\left(s_{1}^{p}\right)_{*}$ and $\varepsilon_{2}$ :

$L_{2}^{P} \rightarrow \operatorname{coker}\left(s_{1}^{p}\right)_{*}$ the canonical morphism, and

(5) $s_{2}^{p}: L_{2}^{p} \rightarrow Z^{p} X$ a lifting of $\varepsilon_{2}: L_{2}^{p} \rightarrow \operatorname{coker}\left(s_{1}^{p}\right)_{*}$.

Given (1)-(5), we define the $R$-dg module $M(p)$ as

$$
M(p)^{i}= \begin{cases}M^{i} & \text { if } i \geq p+1 \\ M^{p}=L_{1}^{p} \oplus L_{2}^{p} & \text { if } i=p \\ o & \text { otherwise }\end{cases}
$$


and the morphism of complexes $s(p): M(p) \rightarrow X$, as

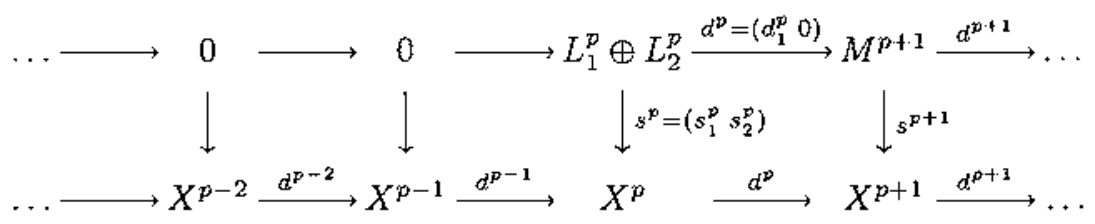

Now, it is straighforward to verify $(a)_{p},(b)_{p}$ and $(c)_{p}$. Finally, we take $M=\underset{p}{\lim } M(p)$.

\section{Minimal objects in bifibred categories}

A bifibred category is a family of categories parametrized by another category, together with a pair of functors (direct and reciprocal images) between the categories of the family, for each morphism of the category of "parametres". To fix our ideas, we may think of the category of modules over any ring. Objects of this category are couples $(R, M)$ where $R$ is a ring and $M$ a $R$-module. Morphisms are also couples $(f, \varphi):(R, M) \rightarrow(S, N)$, where $f: R \rightarrow S$ is a morphism of rings and $\varphi: M \rightarrow f^{*} N$ is a morphism of $R$-modules. We have an evident projection functor $(R, M) \mapsto R$, whose "fibre" over $R$ is the category of $R$-modules: $\operatorname{Mod}(R)$. Finally, for every morphism of rings $f: R \rightarrow S$ we have the well-known functors restriction and extension of scalars

$$
f^{*}: \operatorname{Mod}(S) \longrightarrow \operatorname{Mod}(R) \quad \text { and } \quad f_{*}: \operatorname{Mod}(R) \longrightarrow \operatorname{Mod}(S)
$$

More generally (see [SGA1]),

Definition 3.1. A bifibred category is a functor

$$
P: \mathcal{A} \longrightarrow \mathcal{E}
$$

together with the following structure:

(1) For every $x \in \operatorname{obj} \mathcal{E}$ we call the fibre-category over $x$ the category $\mathcal{A}_{x}$ whose objects are those $a \in \operatorname{obj} \mathcal{A}$ such that $P a=x$ and whose morphisms are those $\varphi \in$ mor $\mathcal{A}$ such that $P \varphi=1_{x}$.

(2) For every $f: x \rightarrow y \in$ mor $\mathcal{E}$ and every $b \in \mathcal{A}_{y}$ we have a cortesian morphism

$$
b^{f}: a \longrightarrow b
$$

which is a $f$-morphisms, i.e., $P\left(b^{f}\right)=f$, determined by the following universal property: for every $f$-morphism $\omega: a^{\prime} \rightarrow b \in \operatorname{mor} \mathcal{A}$, 
there exists a unique morphism of $\mathcal{A}_{x}, \varphi: a^{\prime} \rightarrow a$, such that $b^{f} \varphi=\omega$. Dually, for every $f: x \rightarrow y \in$ mor $\mathcal{E}$ and every $a \in \mathcal{A}_{x}$ we have a cocartesian morphism

$$
a_{f}: a \longrightarrow b
$$

which is a $f$-morphism, such that for every $f$-morphism $\theta: a \rightarrow$ $b^{\prime} \in \operatorname{mor} \mathcal{A}$, there exists a unique morphism of $\mathcal{A}_{y}, \psi: b \rightarrow b^{\prime}$, such that $\psi a_{f}=\theta$.

(3) In the first case, $a$ is called the reciprocal image of $b$ by $f$ and denoted by $f^{*} b$. In the second case, $b$ is called the direct image of $a$ by $f$ and denoted by $f_{*} a$. Both $f^{*} b$ and $f_{*} a$ are unique up to canonical isomorphisms and the definitions are functorial: if $\psi: b \rightarrow b^{\prime} \in \operatorname{mor} \mathcal{A}_{y}$ its reciprocal image $f^{*} \psi$ is the unique morphism of $\mathcal{A}_{x}$ that renders commutative the diagram of $\mathcal{A}$,

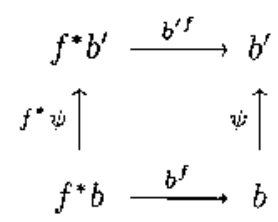

Analogously, we can define the direct image of a morphism $\varphi$ : $a^{\prime} \rightarrow a \in \operatorname{mor} \mathcal{A}_{x}$. These functors

$$
f^{*}: \mathcal{A}_{y} \longrightarrow \mathcal{A}_{x} \quad \text { and } \quad f_{*}: \mathcal{A}_{x} \longrightarrow \mathcal{A}_{y}
$$

are subjected to verify the following conditions: for every pair of morphisms $x \stackrel{f}{\rightarrow} y \stackrel{g}{\rightarrow} z$ of $\mathcal{E}$, there are canonical isomorphisms of functors $f^{*} g^{*}=(g f)^{*}$ and $g_{*} f_{*}=(g f)_{*}$.

Remark 3.2. As an immediate consequerice of the definition of cartesian morphism, every morphism $\omega: a \rightarrow b$ of $\mathcal{A}$ may be factorized in a morphism of the fibre over $P a$ and a cartesian morphism. We will call it the source factorization and denote it by

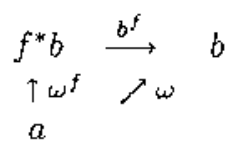

where $f=P \omega$. Obviously, there is a dual factorization (the target factorization) which we will not use in this paper (see [Roig1]). 
Example 3.3. Let $R$ bo a unitary commutative ring, $\operatorname{Adgc}(R)$ the category of $R$-dgc algebras and $\operatorname{Adgc}(R)^{2}$ the category of morphisms of $R$-dgc algebras. The objects of this category are morphisms $\alpha: A \rightarrow B$ of $R$-dgc algebras and the morphisms commutative squares

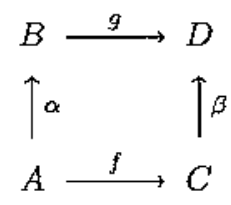

of $\operatorname{Adgc}(R)$. We take as $P$ the domain functor $\operatorname{Adgc}(R)^{2} \rightarrow \operatorname{Adgc}(R)$ which sends $\alpha$ to $A$. Then the fiber categories are the categories of $A$ dgc algebras: $\operatorname{Adgc}(A)=A \backslash \operatorname{Adgc}(R)$. For each morphism of $R$-dgc algebras $f: A \rightarrow C$ we have a reciprocal image functor and a direct image functor

$$
f^{*}: \operatorname{Adgc}(C) \longrightarrow \operatorname{Adgc}(A) \quad \text { and } \quad f_{*}: \operatorname{Adgc}(A) \longrightarrow \operatorname{Adgc}(C)
$$

defined by $f^{*}(\beta)=\beta f$ and $f_{*}(\alpha)={ }_{-} \otimes 1: C \rightarrow C \otimes_{A} B$.

The cartesian morphism $\beta^{f}$ and the cocartesian morphism $\alpha_{f}$ are the commutative squares

$$
\begin{array}{ll}
D \stackrel{1}{\longrightarrow} D & B \stackrel{1 \otimes_{-}}{\longrightarrow} C \otimes_{A} B \\
\uparrow_{\beta f=f^{\cdot}(\beta)} \uparrow_{\beta} \text { and } & \uparrow_{\alpha} \\
A \stackrel{f}{\longrightarrow} C & A \stackrel{f}{\longrightarrow} C
\end{array}
$$

[Roig1] shows how to endow $\mathcal{A}$ with a model structure from given structures on $\mathcal{E}$ and $\mathcal{A}_{x}$. Nevertheless, in order to talk of minimal objects in $\mathcal{A}$ we only need a class of distinguished morphisms, which we define as follows: suppose wc are given classes $S_{\mathcal{E}} \subset \operatorname{mor} \mathcal{E}$ and $S_{x} \subset$ mor $\mathcal{A}_{x}$ for every $x \in \mathrm{obj} \mathcal{E}$ in such a way that they are compatible with reciprocal images; i.e., for every $f: x \rightarrow y \in \operatorname{mor} \mathcal{E}$, one has $f^{*}\left(S_{y}\right) \subset S_{x}$. Then, put

$$
S=\left\{\varphi: a \longrightarrow b \in \operatorname{mor} \mathcal{A} \mid P \sigma \in S_{E} \text { and } \sigma^{P \sigma} \in S_{P_{a}}\right\}
$$

For instance, a cartesian morphism belongs to $S$ if and only if its projection is in $S_{\mathcal{E}}$, and a fibre-morphism $\varphi \in \operatorname{mor} \mathcal{A}_{x}$ belongs to $S$ if and only if it is in $S_{x}$. It is clear that this choice of $S$ agrees with that of weak equivalences made in [Roig1, Theorem 5.1], considering $S_{\mathcal{E}}$ and $S_{x}$ the weak equivalences of the model categories $\mathcal{E}$ and $\mathcal{A}_{x}$, respectively. In particular, if for the category $\operatorname{Adgc}(R)^{2}$ we take $S_{\operatorname{Adgc}(R)}$ and $S_{A}$ the classes of quism of $\operatorname{Adgc}(R)$ and $\operatorname{Adgc}(A)$, respectively, the morphism $(1)$ is in $S$ if and only if $f$ and $g$ are quism. 
Theorem 3.4. Let $m \in \operatorname{obj} \mathcal{A}$. Then, $m$ is a minimal object of $\mathcal{A}$ if and only if $m \in \operatorname{obj} \mathcal{A}_{P m}$ and $P m \in \mathcal{E}$ are minimal objects.

Proof: Let us assume that $m$ is a minimal object of $\mathcal{A}$. Let us show that it is so in $\mathcal{A}_{P m}$. Let $\sigma: a \rightarrow m \in S_{P m}$. Then $\sigma \in S$ and, as $m$ is minimal in $\mathcal{A}$, there exists $\sigma^{\prime}: m \rightarrow a \in$ mor $\mathcal{A}$ such that $\sigma \sigma^{\prime}=1_{m}$. The only thing we have to prove is that $\sigma^{\prime} \in \operatorname{mor} \mathcal{A}_{P m}$ and this follows by evaluating $P$ on both sides of the last equality, which gives us $P \sigma^{\prime}=1_{P m}$.

Let us see that $P m \in \operatorname{obj} \mathcal{E}$ is also a minimal object. Let $s: x \rightarrow$ $P m \in S_{\mathcal{E}}$. Consider the cartesian morphism $m^{s}: s^{*} m \rightarrow m$. It belongs to $S$ because $P\left(m^{s}\right)=s$. Then, since $m$ is minimal in $\mathcal{A}$, there exists $\rho: m \rightarrow s^{*} m$ such that $m^{s} \rho=1_{m}$. Hence $s(P \rho)=1_{P m}$.

Conversely, let $m$ be a minimal object of $\mathcal{A}_{P m}, P m$ a minimal object of $\mathcal{E}$ and $\sigma: a \rightarrow m$ a morphism of $S$. Then $s=P \sigma: P a \rightarrow P m \in S_{\mathcal{E}}$ and, as $P m$ is minimal, there exists $t: P m \rightarrow P a$ such that $s t=1_{P m}$. Let us consider the source factorization of $\sigma: \sigma=m^{s} \sigma^{s}$. Then $\sigma^{s} \in S_{P a}$ and so $t^{*}\left(m^{s}\right): t^{*} a \rightarrow t^{*} s^{*} m=m$ is in $S_{P m}$. Because of the minimality of $m$ in $\mathcal{A}_{P m}$, this morphism has a section $\varphi: m \rightarrow t^{*} a$ and $a^{t} \varphi: m \rightarrow a$ is a section of $\sigma$.

Corollary 1. If for all $x \in \operatorname{obj} \mathcal{E}, \mathcal{A}_{x}$ and $\mathcal{E}$ have enough minimals, then $\mathcal{A}$ has enough minimals.

Proof: Let us build a minimal model for $a \in$ obj $A$ : take a minimal model of $P a$, which exists by hypothesis: $s: m^{\prime} \rightarrow P a$. Take a minimal model of $s^{*} a$ in $\mathcal{A}_{m^{\prime}}: \rho: m \rightarrow s^{*} a$. Then $a^{s} \rho: m \rightarrow a$ is a minimal model for $a$ in $\mathcal{A}$.

Corollary 2. Let $\mathcal{A}$ be endowed with a model category structure such as in [Roig1. Theorem 5.1]. Assume also that, for every $x \in \mathrm{obj} \mathcal{E}, \mathcal{A}_{x}$ and $\mathcal{E}$ have enough minimals. Then the inclusion $\mathcal{A}_{\min } \hookrightarrow \mathcal{A}$ induces an equivalence of categories

$$
\pi \mathcal{A}_{\min } \cong \mathrm{Ho} \mathcal{A}
$$

The following result could also be proved for any suitable category of morphisms directly from the definitions:

Corollary 3. A morphism of $R$-dgc algebras $f: A \rightarrow B$ is a minimal object of $\operatorname{Adgc}(R)^{2}$ if and only if $A$ is a minimal $R$-dgc algebra and $f$ is a minimal morphism.

$\operatorname{Adgc}(R)^{2}$ has enough minimals, for instance, if we take $R$ a zero global dimension ring and restrict ourselves to non-negative homologically connected $R$-dgc algebras (see [Hal, Chapter 9]). A minimal model 
for $f: A \rightarrow B$ in the category of morphisms is constructed as in Corollary 1: we take a minimal model for $A$ in $\operatorname{Adgc}(R), \rho: M_{A} \rightarrow A$, and a minimal model for $f \rho: M_{A} \rightarrow B$ in $\operatorname{Adgc}\left(M_{A}\right)$; that is to say, a commutative triangle of $\operatorname{Adgc}(R)$,

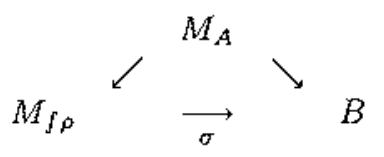

where $\sigma$ is a quism of $R$-dgc algebras. In [Ha1], $M_{A} \rightarrow M_{f \rho}$ is called a $\Lambda$-minimal $\Lambda$-extension.

Dualizing the previous results to right minimal objects and right models, we see that in the category $\Delta^{\circ}$ Set of simplicial sets, taking $S$ to be the morphisms which induces isomorphisms in all the homotopy groups, minimal Kon complexes are the minimal objects in terms of (the dual of) our Definition 1.1. This follows from (the dual of) our Proposition 1.6 and [May, 9.5 and 9.7]. The same proposition and [May, 10.6, 10.7 and 10.13], taking into account that we do not necd the deformation retracts to be strong, show that the minimal Kan fibrations with base $B$ are the minimal objects of $\Delta^{\circ}$ Set $/ B$. These results and the dual of our Theorem 3.2 , give us

Corollary 4. Minimal fibre spaces constitute the minimal objects of $\left(\Delta^{\circ} \text { Set }\right)^{2}$.

Here, $\left(\Delta^{\circ} \text { Set }\right)^{2}$ is the category of morphisms of simplicial sets, bifibred with the codomain functor and minimal fibre space means a minimal Kan fibration $p: E \rightarrow B$ with $B$ a minimal Kan complex. This last corollary could also be deduced from the dual of 1.6 and [May, 10.11 and 10.16].

\section{References}

[B-G] Bousfield, A. K. And Gugenheim, V. K. A. M., "On PL De Rham theory and rational homotopy type," Mem. AMS 179, 1976.

[Ei] Eilenberg, S., Homological dimensions and syzygies, Ann. of Maths. 64 (1956), 328 -336.

[G-Z] Gabriel, P. AND Zisman, M., "Calculus of fractions and homotopy theory," Springer, 1967.

[H-T] Halperin, S. AND TANRÉ D., Homotopie filtrée et fibrés $C^{\infty}$, Ill. J. of Maths. 34 (1990), 284-324. 
[Hart] Hartshorne, R., "Residues and duality," Springer LNM 20, 1966.

[Hal] HALPERIN, S.. "Lectures on minimal models," Mem. SMF, nouvelle série, 9/10, 1983.

[Kan] KAN, D., Minimal frec CSS groups, $I l l$. J. of Maths. 2 (1958), 449-476.

[Mat] MatsumlRA, H., "Commutative ring theory," Cambridge University Press, 1986.

[May] MAY, J. P., "Simplicial objects in algebraic topology," Van Nostrand, 1967.

[Qui] QUILı,EN, D. G., "Homotopical algebra," Springer LNM 47, 1967.

[Roig1] Rorg A., Model category structures in bifibred categories, to appear in $J$. of Pure and Applied Algebra.

[Roig2] RoIg, A., Formalizability of $D G$ modules and morphisms of $D G C$ algebras, to appcar in $I l l . J$. of Maths.

[Roig3] RoIG, A., Modèles minimaux et foncteurs dérivés, to appear in $J$. of Pure and Applied Algebra.

[SGAI] GRothendieck, A., "Séminaire de Géometrie Algébrique 1," Springer LNM 224, 1971.

[Tate] TAte, J., Homology of noctherian rings and local rings, $I l l . J$. of Maths. 1 (1957), 14--27.

Departament de Matemàtica Aplicada I, ETSEIB

Lniversitat Politècnica de Catalunya

Av. Diagonal 647

08028 Barceloná

SPAIN

Primera versió rebuda el 29 de Setembre de 1992, darrera versió rebuda el 8 de Goner de 1993 